\title{
Photoconductivity and the evolution of energy bands in fluid xenon
}

\author{
R. Reininger, U. Asaf, and I. T. Steinberger \\ Racah Institute of Physics, The Hebrew University, Jerusalem 91904, Israel \\ V. Saile \\ Hamburger Synchrotronstrahlungslabor (HASYLAB), Deutsches Elektronen-Synchrotron (DESY), \\ D-2000 Hamburg 52, Federal Republic of Germany \\ P. Laporte \\ Equipe de Spectroscopie, Laboratoire (No. 171) associé au Centre National, \\ de la Recherche Scientifique 158 bis cours Fauriel, F-42023 Saint Etienne Cedex, France
}

(Received 17 November 1982)

\begin{abstract}
Photoconductivity excitation spectra for densities above $10^{21}$ atoms $/ \mathrm{cm}^{3}$ up to the triple point exhibit a threshold that moves to lower photon energies with increasing density. Its value extrapolated to zero density is $11.10 \mathrm{eV}$, i.e., the difference between the energy minimum of the $\mathrm{Xe}_{2}{ }^{+}$molecular ion and the ground-state energy of the xenon atom. The hole polarization energy $P^{+}$was evaluated using the Born charging energy for the same density range. These results along with the previously determined values of the electron affinity $V_{0}$ yielded a full representation of the energy-band edges for the whole density range. The relationship between these results and those on the evolution of excitons is discussed in detail.
\end{abstract}

\section{INTRODUCTION}

In a recent publication, ${ }^{1}$ we described results on photoresponse in xenon at densities below $\sim 10^{21}$ atoms $/ \mathrm{cm}^{3}$. In this density range the photoelectric current is predominantly due to photoionization even for photon energies less than the ionization energy of the xenon atom $(12.13 \mathrm{eV})$. The photoionization takes place in two steps: Upon absorption of a photon the xenon excimer $\mathrm{Xe}_{2}{ }^{*}$ is formed, followed by spontaneous ionization of the excimer. This involves the formation of the xenon molecular ion $\mathrm{Xe}_{2}{ }^{+}$and a free electron. This essentially atomic process, modified by molecular interactions, is characterized by an excitation spectrum rich in features, especially at the lowest densities. The process is possible only above the photon energy threshold of $11.10 \mathrm{eV}$ that is given by the energy differences of the ground state of the molecular ion and the ground state of the free atom. In contrast, the present study deals with higher densities where the photoelectric current is due to photoconductance, its excitation spectrum is almost featureless and may extend to photon energies considerably less than $11.10 \mathrm{eV}$, depending on the density.

The main facts associating the photoelectric current for number densities $\rho_{N}>10^{21}$ atoms $/ \mathrm{cm}^{3}$ with photoconduction are as follows. (a) A photoresponse threshold $E_{\mathrm{pc}}$ is observed in liquid xenon at the triple point very near to the photoconductivity threshold of the solid. Its position is consistent with predictions on the change of the band gap based upon the density change at the phase transition. ${ }^{2}$

(b) The electron mobility ${ }^{3} \mu$ of liquid xenon near the triple point is very high, namely 2200 $\mathrm{cm}^{2} / \mathrm{V} \mathrm{sec}$. Its value is about the half of that in solid xenon; this is understood on the basis of the density and compressibility change at the phase transition. ${ }^{3}$

(c) Reflection bands due to excitons appear both in solid and liquid xenon. ${ }^{4}$ Band gaps calculated from Wannier exciton series are in close agreement with those determined from the photoconductivity threshold. These facts imply that near the triple point the photoresponse has to be, indeed, attributed to photoconduction proper. For lower densities (down to about $10^{21}$ atoms $/ \mathrm{cm}^{3}$ ) the dominance of photoconduction can be surmised from the continuous variation of the energy $V_{0}$ of the quasifree electron. ${ }^{6}$ Clear evidence that this is, indeed, the case will be presented in this paper.

The exciton bands in fluid xenon were studied for a wide range of densities. ${ }^{7,8}$ They were found to be entities different from atomic lines broadened and shifted by molecular interactions, with each exciton 
band appearing only above its own characteristic density. Since excitons are closely related to the electron band structure, the above facts raise the question whether there exists a definite threshold for conduction in a solidlike energy band as well. We note in this context that the zero-field electron mobility $\mu_{0}$ in fluid xenon has a rather deep minimum ${ }^{5}$ at a density of about $4 \times 10^{21}$ atoms $/ \mathrm{cm}^{3}$. This might be interpreted on the basis of the above considerations by regarding the mobility minimum as the threshold for conduction in a solidlike conduction band.

Recent systematic photoinjection studies in fluid xenon, ${ }^{6}$ krypton, ${ }^{6}$ and argon ${ }^{9}$ gave direct evidence on the energy $V_{0}$ of a conduction-band electron (in other words, the electron affinity) as a function of density. The results showed that the value of $V_{0}$ changes continuously and gradually from densities of that of a dilute gas to that of a triple-point liquid, with a minimum in between. Moreover, preliminary results ${ }^{10}$ on the density dependence of the photoconductivity band gap $E_{\mathrm{pc}}$, referring to photon energies below $10.2 \mathrm{eV}$ and densities above $4 \times 10^{21}$ atoms $/ \mathrm{cm}^{3}$ indicated that $E_{\mathrm{pc}}$ varies linearly with density within this range. These facts seem to contradict those quoted above concerning the excitons. The study of photoconductivity excitation spectra in a broad range of densities was undertaken in order to learn whether there is, at any density, a clear threshold for the appearance of a solidlike conduction band or else such conduction develops continuously from conduction in the gas phase. In fact, the experimental results indicated that the development is, indeed, continuous.

The paper also correlates data on the mobility and on the evolution of excitonic bands with the results

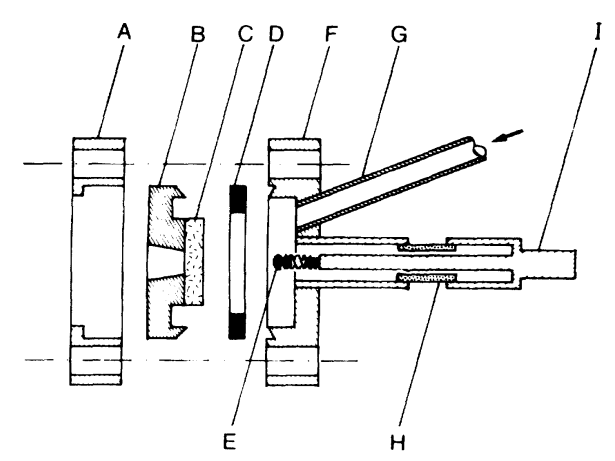

FIG. 1. Cross section of the sample cell. $A$ : retaining ring; $B$ and $F$ : parts of "Conflat" seal; $C$ : $\mathrm{LiF}$ window, glued to $B ; D$ : copper gasket; $E$ : spring and contact head; $G$ : filling tube; $H$ : ceramic insulator of ultrahigh-vacuum feedthrough; $I$ : central stalk welded into the feedthrough. The cell has two feedthroughs, but for simplicity only one is drawn. on $E_{\mathrm{pc}}$. Finally, the energies of the bottom of the conduction band $V_{0}$, the top of the valence band $V_{0}-E_{\mathrm{pc}}$, the hole polarization $P^{+}$, and $V_{0}-E_{\mathrm{pc}}+P^{+}$are presented as a function of the density, for the first time for any nonmetal.

\section{RESULTS}

The photoconductivity excitation spectra were obtained using monochromated synchrotron radiation from the DORIS storage ring at DESY. The experimental arrangement has been described before ${ }^{1,10,11}$ except for the details of the sample cell: it is presented in Fig. 1. The cell was constructed of stainless steel with a copper gasket. The $\mathrm{LiF}$ window was glued to one-half of a Conflat seal by means of a low-vapor-pressure epoxy resin. For application at low temperatures, a further ring, made of duralumin, served as substrate for the window, being glued on one side of the Conflat seal and to the window on the other. This ring is not shown in the figure. The inner side of the window accommodated two intertwined gold electrodes prepared by sputtering. Adjacent prongs of the electrodes were spaced at $0.36 \mathrm{~mm}$ from each other. High-voltage ultrahigh-vacuum feedthroughs with an interior stainless-steel stalk, spring and polished pinhead ensured electrical contact to the gold electrodes.

In the overlapping density range and spectral region these spectra are in full accord with those obtained earlier employing as light source the continuum of a Tanaka lamp filled with krypton or argon. Reflection spectra of the $\mathrm{MgF}_{2}$-fluid-xenon interface were taken near normal incidence using conventional sources, by methods described earlier. ${ }^{4,12}$



FIG. 2. Normalized photocurrent as a function of the energy of incident photons at various number densities $\left(10^{21}\right.$ atoms $\left./ \mathrm{cm}^{3}\right)$ and temperatures (in $\left.\mathrm{K}\right)$, respectively: $a$, $10.9,242.3 ; b, 8.3,284.3 ; c, 6.2,296.3 ; d, 4.1,296.5 ; e$, $3.3,295.0$; and $f, 2.3,296.5$. 
Figure 2 represents the photocurrent $i_{\mathrm{ph}}$ at a fixed voltage as a function of the photon energy for a set of densities. The currents were normalized at each wavelength to the number of photons incident on the xenon gas or fluid per unit time; this number was established by recording the transmission of the $\mathrm{LiF}$ window at various temperatures as well as the output flux of the HONORMI monochromator.

The main feature common to all densities is the onset of photocurrent at a certain photon energy threshold and its persistence at all higher photon energies. The threshold shifts continuously to higher photon energy with decreasing density and simultaneously the initial slope becomes smaller. ${ }^{10}$ Crossing the critical isotherm (between graphs $c$ and $d$ ) has no effect. At the lowest density presented (curve $f$ ) the current level rises considerably again at about $11.0 \mathrm{eV}$ : the broad band seen there (and, less clearly, on curve $e$ as well) is caused by the atomicmolecular photoionization effects. ${ }^{1}$ At the higher densities (curves $a-d$ ) the band is submerged in the higher and broader photoconductivity excitation spectra.

The apparent structure around $8.8 \mathrm{eV}$ is not fully reproducible. At or near this photon energy there is no structure in the reflection spectrum at any density (see Fig. 3), the sample is almost totally transparent and therefore the peak may be associated with photoemission from the rear part of the cell or with impurity photoionization or photoconduction in the sample; the impurities might have originated, e.g., from the epoxy resin used to mount the window. In any case there is no doubt that this peak is not intrinsic to the properties of fluid xenon and therefore it will not be discussed further.

More details of the photocurrent excitation spectra can be understood if these spectra are compared with the corresponding reflectivity spectra. Figure 3 shows the results of such a comparison for an intermediate density $\left(6.2 \times 10^{21}\right.$ atoms $\left./ \mathrm{cm}^{3}\right)$. (The reflec-

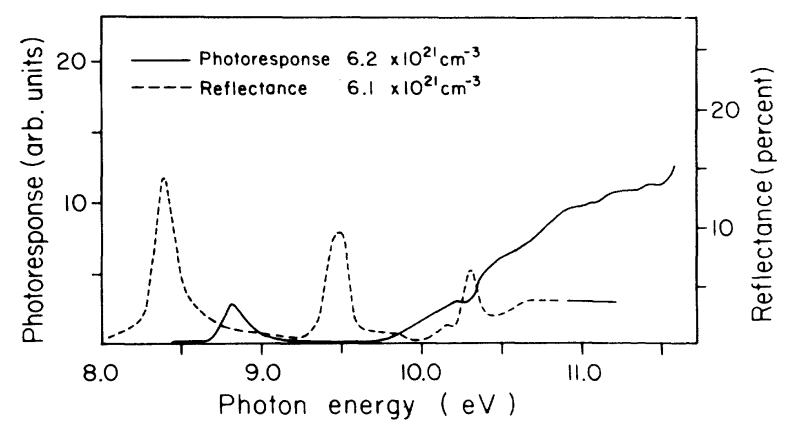

FIG. 3. Normalized photocurrent compared with the reflectance of the xenon- $\mathrm{MgF}_{2}$ interface at similar densities. tivities were measured in Saint Etienne with the cooperation of J. L. Subtil.) The reflection spectrum exhibits the $n=1\left[\Gamma\left(\frac{3}{2}\right]\right.$ and $n^{\prime}=1\left[\Gamma\left(\frac{1}{2}\right)\right]$ excitonic peaks at 8.4 and $9.45 \mathrm{eV}$, respectively. The small peak at $10.16 \mathrm{eV}$ can be attributed to transition to the $5 d\left(\frac{5}{2}\right)_{3}^{0}$ level, forbidden in the dilute gas. The peak at $10.32 \mathrm{eV}$ is a band that has developed from the two neighboring $5 d\left(\frac{3}{2}\right)_{1}^{0}$ and $7 s\left(\frac{3}{2}\right)_{1}^{0}$ states. The reflectivity graph does not extend beyond $10.8 \mathrm{eV}$, since the measurements were primarily designed to study the evolution of the three lowest-energy exciton bands and therefore a $\mathrm{MgF}_{2}$ front window was employed. The $n=2\left[\Gamma\left(\frac{3}{2}\right)\right]$ Wannier exciton around $9.0 \mathrm{eV}$ is not observed in this graph since this exciton appears ${ }^{8}$ only for densities above $10.8 \times 10^{21}$ atoms $/ \mathrm{cm}^{3}$. Turning now to the corresponding photocurrent excitation spectrum, it should be noted that the reflectivity maximum at $10.32 \mathrm{eV}$ is associated with a decrease in the photocurrent. This feature can be attributed to competition for the incident photons between the band-toband transitions causing photoconductivity and the discrete transition at $10.32 \mathrm{eV}$. While for the interband transitions the electrons and holes become quasifree and are separated by the electric field applied, a discrete bound state most probably has alternative decay channels like luminescence, thus reducing the measured photocurrent at the line. A simple correction taking into account the losses due to the reflectivity at the lines cannot account for the depths of the minima in the photocurrent spectra.

The dip at about $11.6 \mathrm{eV}$ in the photoconductivity excitation spectra (Figs. 2 and 3 ) is caused by the $5 d^{\prime}\left(\frac{3}{2}\right)_{1}^{0}$ atomic peak, broadened and shifted due to molecular interactions. It should also be noted that the peak of the $n^{\prime}=1\left[\Gamma\left(\frac{1}{2}\right)\right]$ exciton at about 9.45 $\mathrm{eV}$ is very close to the threshold for the highestdensity graph $a$ of Fig. 1 and therefore the shape of the threshold is probably modified for this density. At still higher liquid densities and in the solid the 9.45-eV discrete reflection peak appears as a pronounced dip in the photoconductivity excitation spectrum. ${ }^{2,10}$

Comparison of the reflection spectra with the photoconductivity excitation spectra reveals another interesting point: The reflection peaks due to excitonic bands and the atomic lines modified by molecular interactions change in position very little with density, ${ }^{7,8}$ while the photoconductivity threshold changes considerably, moving to higher photon energies and decreasing in slope with decreasing density. The latter facts were stressed when discussing Fig. 2; the former one is also clearly illustrated in the same figure by the invariance of the position of the dip at $10.3 \mathrm{eV}$. 
The structure in the photoconductivity excitation spectrum at and around $10.3 \mathrm{eV}$ may cause a misinterpretation of the photoconductivity excitation spectra for densities where the photoconduction threshold is very near to $10.3 \mathrm{eV}$. For example, curve $d$ of Fig. 2 may be interpreted as involving two thresholds: a gradual one at about $10 \mathrm{eV}$ and a much steeper one at about $10.35 \mathrm{eV}$ as was done in a preliminary report of these results. ${ }^{13}$ Two arguments against a two-threshold interpretation are (a) the position and width of the dips is very close to that of the corresponding reflection bands (Fig. 2), and (b) a continuous curve with a continuously varying derivative is obtained for the density dependence of the photoconductivity threshold (Fig. 4).

Usually, $E_{\mathrm{pc}}$ is determined from graphs of the kind presented in Fig. 2 by the intercepts of extrapolated straight portions of a power law: $i_{\mathrm{ph}}^{m}$ vs $E, m$ being mostly chosen to be either $\frac{1}{2}, 1$, or 2 in accord with some theoretical model, but often somewhat arbitrarily. On the other hand, defining $E_{\mathrm{pc}}$ as the start of the rising portion of the $i_{\mathrm{ph}}-\mathrm{vs}-h v$ graph has an intrinsic element of inaccuracy, especially if the noise level of the zero line is not negligible (e.g., graphs $e$ and $f$ in Fig. 2). For the data of Fig. 4, the photocurrent thresholds were determined by the latter method, taking the averages of two independent determinations. Very similar results were obtained if $E_{\mathrm{pc}}$ was determined from the intercept of the linear part of $i_{\mathrm{ph}}^{1 / 2}$-vs- $h v$ plots. It is seen that at the highest densities $E_{\mathrm{pc}}=9.19 \mathrm{eV}$. Previous determinations of the photoconductivity threshold in liquid xenon gave $E_{\mathrm{pc}}=9.20 \mathrm{eV}$. 20 The increase of $E_{\mathrm{pc}}$ with decreasing density is seen to be monotonous, almost linear, down to a density ${ }^{10}$ of about $4 \times 10^{21}$ atoms $/ \mathrm{cm}^{3}$, and having a faster-rising portion at the lowest densities. $E_{\mathrm{pc}}$ extrapolates to $11.10 \mathrm{eV}$ at zero density. This limit is equal to the difference between the energy minimum of the xenon molecular ion $\mathrm{Xe}_{2}{ }^{+}$and the xenon atom ground state. $^{1}$ Transitions between the two states were shown to be possible ${ }^{1}$ for all photon energies above

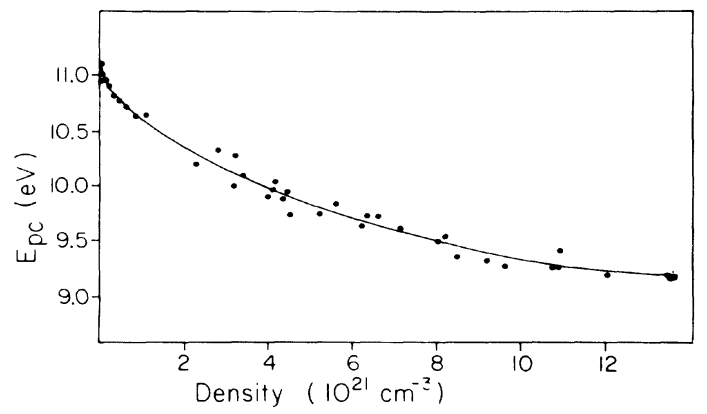

FIG. 4. The density dependence of the photoconductivity threshold.
$11.10 \mathrm{eV}$ due to the Hornbeck-Molnar process ${ }^{14}$ via an excimer state $\mathrm{Xe}_{2}{ }^{*}$, provided the density is larger than about $10^{19}$ atoms $/ \mathrm{cm}^{3}$.

In order to complete the picture on the evolution of the electron energy bands with density, the polarization energy of the valence-band hole $P^{+}$was evaluated as a function of the number density $\rho_{N}$. Following Messing and Jortner's work ${ }^{15}$ on doped fluid argon, the simple Born charging energy formula was used,

$$
P^{+}=-\frac{e^{2}}{2 \sigma}\left(1-\frac{1}{\epsilon}\right),
$$

where $e$ is the electron charge, $\sigma=0.39 \mathrm{~nm}$ is the calculated hard-core radius of a xenon atom, ${ }^{16}$ and $\epsilon$ is the long-wavelength optical dielectric constant. Near the triple point the value of $\epsilon$ determined directly ${ }^{17}$ was used; at other densities it was calculated using the triple-point value and the ClausiusMossotti formula. The use of the simple formula follows considerations by Messing and Jortner ${ }^{15}$ who showed that the results of Eq. (1) for Xe-doped fluid argon are in accord within $\pm 10 \%$ with results obtained by means of Lekner's screening function, ${ }^{18}$ taking into account values of pair correlation functions and the Kirkwood superposition approximation $^{19}$. The relevance of both determinations of $P^{+}\left(\rho_{N}\right)$ may be inferred by comparing of directly measured values ${ }^{9}$ of the quasifree electron energy $V_{0}\left(\rho_{N}\right)$ in fluid argon with values based on the calculated $P^{+}\left(\rho_{N}\right)$ values and spectroscopic evidence. ${ }^{15}$ $P^{+}\left(\rho_{N}\right)$ as evaluated by means of Eq. (1) appears in Fig. 5; it is seen that $P^{+}=0$ for $\rho_{N}=0$ and that $P^{+}\left(\rho_{N}\right)$ is an almost linear function. The highest value of $\left|P^{+}\right|$obtained at the triple-point density, is about $0.9 \mathrm{eV}$. However, estimates of $\left|P^{+}\right|$for solid xenon, obtained by different methods, yielded considerably higher values: $1.32 \mathrm{eV}$ (Ref. 20) and $1.39 \mathrm{eV}$ (Ref. 21), respectively. The discrepancy is too large to attribute it to the change in $\epsilon$ due to the density change upon the phase transition. This effect would increase $\left|P^{+}\right|$as determined from Eq. (1) only by about $10 \%$. Thus it is possible that the

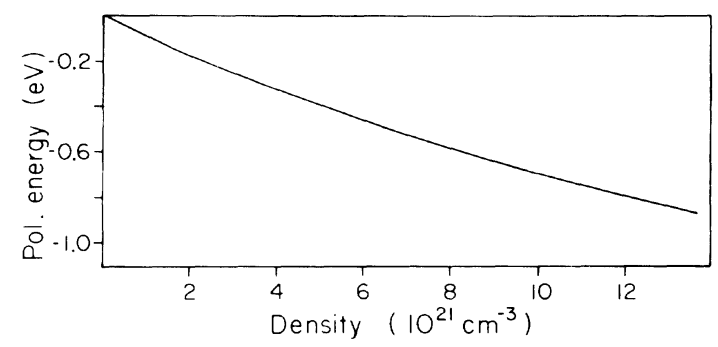

FIG. 5. The calculated density dependence of the hole-polarization energy $P^{+}$. See text. 


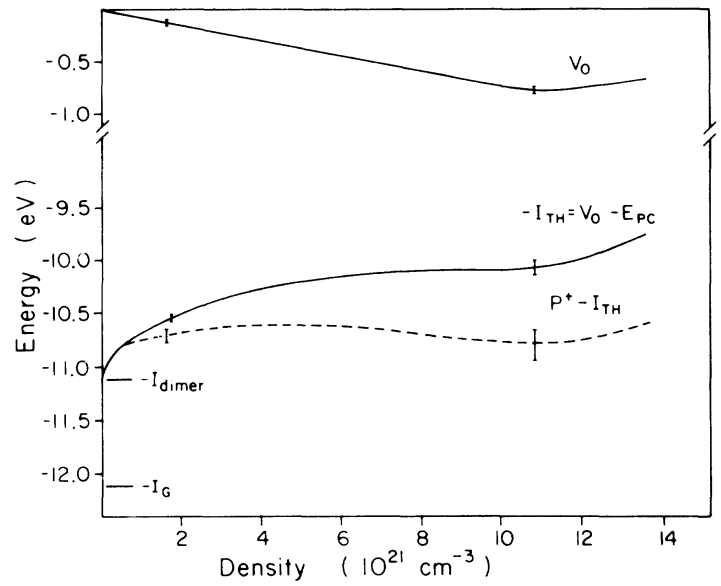

FIG. 6. Density dependence of energy-band parameters.

true values of $\left|P^{+}\right|$are appreciably higher than indicated in Fig. 5.

Figure 6 summarizes the evolution of the band structure in fluid xenon on the basis of the above results. The zero line is the vacuum level. $V_{0}$ is the energy of the free-electron state at the bottom of the conduction band (or the electron affinity), reproduced from a previous publication. ${ }^{6}$ The ionization energy of an electron at the top of the valence band

$$
I_{\mathrm{TH}}=-V_{0}+E_{\mathrm{pc}}
$$

is seen to decrease with increasing density, though it stays roughly constant for densities between 5 and $10 \times 10^{21}$ atoms $/ \mathrm{cm}^{3}$. The graph also includes $P^{+}-I_{\mathrm{TH}}$. This would be the ground state of a valence-band electron if there were no holepolarization effects as expressed by $P^{+}$. Apart from an initial rise at low densities, this level seems to stay constant (within the experimental accuracy) for a wide density range. Apparently the results presented in Fig. 6 are the first case of any nonmetal, crystalline or not, where the evolution of these band-structure parameters was followed in detail.

\section{DISCUSSION}

\section{A. Photoconductivity; electron mobility and excitons}

The similarity of values of several band-structure parameters in solid xenon and in the corresponding liquid has been stressed repeatedly. ${ }^{2,4}$ The continuous and gradual change of the level of the bottom of the conduction band, the level of the top of the valence band, and the hole-polarization energy $P^{+}$ with density found in this work indicated that there is no sudden change in the energy levels relevant to the electrical conduction. This is true down to densities of the order of $10^{21}$ atoms $/ \mathrm{cm}^{3}$. Below this density there is a marked increase in the slope $\left|d E_{\mathrm{pc}} / d \rho_{N}\right|$, while $V_{0}$ is already very small. This indicates the formation of a valence band in this density range; the main contribution to the current is due to the Hornbeck-Molnar process. ${ }^{1}$ For the processes involved in electron transport, in the whole range, other information has to be invoked. The present results will now be compared with the density dependence of the electron mobility ${ }^{5}$ and of the exciton bands. ${ }^{7,8}$

It was demonstrated by Huang aild Freeman ${ }^{5}$ in their work on the electron mobility of fluid xenon that the product of the number density by the zerofield mobility $\mu_{0} \rho_{N}$ is constant from zero density up to about $\rho_{N}=3 \times 10^{20}$ atoms $/ \mathrm{cm}^{3}$. This is a typical behavior of the zero-field mobility in a dilute gas. Above this density there is a minimum in $\mu_{0} \rho_{N}$ (at $\sim 4 \times 10^{21}$ atoms $/ \mathrm{cm}^{3}$ ), attributed to ${ }^{5}$ quasilocalization due to density fluctuations in the liquid. This minimum can be eliminated by a slight increase of the temperature, indicating that the potential well involved in the localization is very shallow. The mobility is strongly dependent on the applied electric field $F$, but at any given field $F, \mu(F)$ varies slowly with density without sudden changes; $\mu(F)$ always has a minimum at about $\rho_{N} \sim 4 \times 10^{21}$ atoms $/ \mathrm{cm}^{3}$, i.e., near the critical density.

The excitonic bands in fluid xenon (and krypton) behave differently. The $n^{\prime}=1\left[\Gamma\left(\frac{1}{2}\right)\right]$ exciton appears only at densities above $2.7 \times 10^{21}$ atoms $/ \mathrm{cm}^{3}$; the $n=1\left[\Gamma\left(\frac{3}{2}\right)\right]$ only above $4.9 \times 10^{21}$ atoms $/ \mathrm{cm}^{3}$, and the $n=2\left[\Gamma\left(\frac{3}{2}\right)\right]$ exciton only above $10.8 \times 10^{21}$ atoms $/ \mathrm{cm}^{3}$. In comparison, we note again that $\mu_{0} \rho_{N}$ deviates $^{5}$ from its constant value of $\rho_{N} \sim 3 \times 10^{20}$ atoms $/ \mathrm{cm}^{3}$. Thus the exciton threshold densities are considerably above those which indicate a deviation from simple "gaslike" conduction. A detailed study $^{7}$ of the evolution of the $n=1\left[\Gamma\left(\frac{3}{2}\right)\right]$ exciton indicated that for the appearance of such excitons light absorption has to take place in a cluster of at least 10 atoms occupying not more than a volume of $1.5 \mathrm{~nm}^{3}$. If some similar condition would be necessary for solidlike conduction in an energy band as well, a rather sudden change of $V_{0}$ and $E_{\mathrm{pc}}$ could be expected at a density, where the individual clusters do not overlap sufficiently to form a conducting bridge between the electrodes. In other words, one might expect percolation effects at some density. The results of this work show no such effects.

The different behavior of excitons and of electrons contributing to conduction can be readily understood following arguments by Basak and Cohen, ${ }^{22}$ leading to their multiple-scattering theory 
of mobility in fluid rare gases. The de Broglie wavelength $\lambda_{e}$ for a thermalized electron at $T=300 \mathrm{~K}$ turns out to be about $6 \mathrm{~nm}$, if one assumes Boltzmann equipartition and thermal electron velocities. It was pointed out ${ }^{22}$ that as long as the mean free path is large compared with $\lambda_{e}$ and $\lambda_{e}$ is much larger than the average interatomic distance $a$, conduction electrons are not scattered by individual atoms, but rather by density fluctuations. For $\rho_{N}=10^{21}$ atoms $/ \mathrm{cm}^{3}, a=1 \mathrm{~nm}$, so $\lambda_{e} / a=6$; for all other densities discussed in this paper the ratio is even larger (up to $\sim 14$ near the triple point). Because of the large value of $\lambda_{e}$, the thermal or almost-thermal conduction electron cannot be confined to a small cluster (typical dimension: $\sim 1 \mathrm{~nm}$ ), that is, indeed, capable of accommodating an exciton. Thus the energy at which the electron finds itself within the sample after it had been excited (i.e., $\left.V_{0}\right)$ is determined by properties averaged over many atoms. The transition to gaslike conduction, characterized by single scattering on individual atoms, occurs gradually for $\rho_{N}<10^{21}$ atoms $/ \mathrm{cm}^{3}$, as the interatomic distance becomes comparable to $\lambda_{e}$.

Confinement of an exciton to a cluster does not constitute, of course, any difficulty as the exciton energy is such as to ensure a $\lambda_{e}$ value consistent with the electron orbit.

\section{B. Band gaps and the position of discrete transitions}

Another interesting point of comparison between bound and quasifree electrons is to consider the relative positions of excitonic and modified atomic peaks in comparison with the photoconductivity threshold $E_{\mathrm{pc}}$. $E_{\mathrm{pc}}$ is equal to the energy difference between the bottom of the conduction band $\left(V_{0}\right)$ and the top of the uppermost valence band $\left(V_{0}-E_{\mathrm{pc}}\right)$. The $\Gamma\left(\frac{3}{2}\right)$ exciton series is formed by the association of a conduction-band electron with a hole from this topmost valence band. Figure 4 showed that $E_{p c}$ varies considerably in the density range of 4.9 to $13.5 \times 10^{21}$ atoms $/ \mathrm{cm}^{3}$, in which the $n=1\left[\Gamma\left(\frac{3}{2}\right)\right]$ exciton discussed exists. In the same range there is, however, very little change in the position of the excitonic peak. $^{8}$ Such behavior was predicted by W.B Fowler ${ }^{20}$ for excitons of small radius; according to his considerations, electronic polarization can be neglected for cases of excited states (excitons, $F$ centers, etc.), where the average radius of the orbit is considerably less than the interatomic distance.

\section{Relations between band-structure parameters and ionization potentials}

It was stated above in the experimental section that the calculated values of the hole-polarization energy $\mathrm{P}^{+}$of Fig. 5 are smaller in absolute value than those evaluated by other methods. ${ }^{20,21}$ It is impossible to tell with certainty which calculations are the correct ones, but in any case there would be little difference in the general trend of these graphs.

Figure 6 shows that the energy of the top of the valence band $-I_{\mathrm{TH}}=V_{0}-E_{\mathrm{pc}}$ stays constant for a broad range of densities and the same is true about the energy $P^{+}-I_{\mathrm{TH}}$. This latter energy level is the ground state of a valence-band hole that would be obtained if there was no hole-polarization energy $P^{+}$. Both $-I_{\mathrm{TH}}$ and $P^{+}-I_{\mathrm{TH}}$ change considerably at low densities (below $3 \times 10^{21}$ and $1 \times 10^{21}$ atoms $/ \mathrm{cm}^{3}$, respectively). It is tempting to relate these changes to valence-band formation from the $5 p^{6}$ atomic cores with increasing density. It is seen in Fig. 6 that the low-density limit of both $-I_{\mathrm{TH}}$ and $P^{+}-I_{\mathrm{TH}}$ is very near to the adiabatic ionization potential of the xenon $\operatorname{dimer}^{23} I_{\text {dimer }}=11.13 \mathrm{eV}$. This fact is at first surprising, since the FranckCondon principle would make very improbable direct transitions from the free atoms (or from the dimers) into the minimum of the configurational curve of the molecular ion, occurring at the rather close distance of $0.285 \mathrm{~nm}^{23}$ However, we showed in Ref. 1 that such a transition is, indeed, very probable for densities higher than $10^{19}$ atoms $/ \mathrm{cm}^{3}$ and for all photon energies larger than the threshold $I_{\text {dimer }}-D\left(\mathrm{Xe}_{2}\right), D\left(\mathrm{Xe}_{2}\right)$ being the dissociation energy $\left[D\left(\mathrm{Xe}_{2}\right)=0.024 \mathrm{eV}\right.$ (Ref. 24)] of the Van der Waals molecule $\mathrm{Xe}_{2}$. This is so because the HornbeckMolnar process appears for both at the maxima and in the wings of the atomic absorption lines. The ionization limit of the free atom $I_{G}=12.127 \mathrm{eV}$ would thus become effective only at extremely low densities. Now the Hornbeck-Molnar process deals, in fact, with interactions of the excited states only: $I_{\text {dimer }}-D\left(\mathrm{Xe}_{2}\right)$ would be an upper limit for the ionization energy even if there were no changes in the ground state, i.e., no polarization by the hole. In other words, $P^{+}-I_{\mathrm{TH}}$ must be equal to or smaller than $I_{\text {dimer }}-D\left(\mathrm{Xe}_{2}\right)$, in accord with the results presented here.

\section{ACKNOWLEDGMENTS}

The authors wish to express their thanks to several institutions (DESY, Deutscher Akademischer Austauschdienst and the Israel Commission for Basic Research) for their contributions towards the success of this project. Among numerous individuals we specially thank Dipl.-Phys. W. Jark, of Hamburg University, for valuable technical help. 
${ }^{1}$ P. Laporte, V. Saile, R. Reininger, U. Asaf, and I. T. Steinberger, Phys. Rev. A (in press).

${ }^{2}$ U. Asaf and I. T. Steinberger, Phys. Rev. B $\underline{10}, 4464$ (1974).

${ }^{3}$ L. S. Miller, S. Howe, and W. E. Spear, Phys. Rev. 166, 871 (1968).

${ }^{4}$ I. T. Steinberger and U. Asaf, Phys. Rev. B $\underline{8}, 914$ (1973).

${ }^{5}$ S. S. S. Huang and G. R. Freeman, J. Chem. Phys. $\underline{68}$, 1355 (1978).

${ }^{6}$ R. Reininger, U. Asaf, and I. T. Steinberger, Chem. Phys. Lett. 90, 287 (1982).

${ }^{7}$ P. Laporte and I. T. Steinberger, Phys. Rev. A 15, 2538 (1977).

${ }^{8}$ P. Laporte, J. L. Subtil, U. Asaf, I. T. Steinberger, and S. Wind, Phys. Rev. Lett. 45, 2138 (1980).

${ }^{9}$ R. Reininger, U. Asaf, I. T. Steinberger, and S. Basak Phys. Rev. B (in press).

${ }^{10}$ R. Reininger, U. Asaf, P. Laporte, and I. T. Steinberger, in Proceedings of the Seventh International Conference on Conduction and Breakdown in Dielectric Liquids, edited by W. F. Schmidt (Springer, Berlin, 1981), p. 69; J. Electrostatics 12, 123 (1982).
${ }^{11}$ V. Saile, G. Gürtler, E. E. Koch, A. Kozevnikov, M. Skibowski, and W. Steinmann, Appl. Opt. 15, 2559 (1976).

12P. Laporte, J. L. Subtil, M. Bon, and H. Damany, Appl. Opt. 20, 2133 (1981).

${ }^{13}$ R. Reininger, U. Asaf, P. Laporte, V. Saile, and I. T. Steinberger, Bull. Israel Phys. Soc. 28, 21 (1982).

14J. A. Hornbeck and J. P. Molnar, Phys. Rev. $\underline{84}, 621$ (1951).

15I. Messing and J. Jortner, Chem. Phys. 24, 183 (1979).

${ }^{16}$ N. W. Ashcroft, Physica (Utrecht) $\underline{35}, 148$ (1967).

${ }^{17}$ A. C. Sinnock and B. L. Smith, Phys. Rev. 181, 1297 (1969).

18J. Lekner, Phys. Rev. 158, 130 (1967).

${ }^{19}$ J. G. Kirkwood, J. Chem. Phys. $\underline{3}, 300$ (1935).

${ }^{20}$ W. B. Fowler, Phys. Rev. 151, 657 (1966).

${ }^{21}$ L. E. Lyons and M. G. Sceats, Chem. Phys. Lett. ㅁ, 217 (1970).

${ }^{22}$ S. Basak and M. H. Cohen, Phys. Rev. B 20, 3404 (1979).

${ }^{23}$ C. Y. Ng, D. J. Trevor, B. H. Mahan, and Y. T. Lee, J. Chem. Phys. 65, 4327 (1976). 